\title{
Desarrollo de criterios normativos y protocolos de seguridad para experimentación con cohetería dentro del territorio colombiano*
}

| Fecha de recibido: 30 de abril del 2020 | Fecha de aprobación: 23 de septiembre del 2020 |

\section{Juan Sebastián Bonilla Romero \\ Estudiante de Ingeniería Aeronáutica (en proceso de grado) \\ Universidad de San Buenaventura \\ Grupo de investigación Aerotech \\ Rol de investigador: teórico \\ https://orcid.org/0000-0002-3681-1484 \\ $\bowtie$ grjuan@academia.usbbog.edu.co}

José Alejandro Urrego Peña

Ph. D. en Ingeniería Mecánica

Universidad de San Buenaventura

Grupo de investigación Aerotech

Rol de investigador: teórico y experimental

https://orcid.org/0000-0001-9823-5091

$\bowtie$ jurrego@usbbog.edu.co

\author{
Rubén Salazar \\ Magíster en Ingeniería Aeroespacial \\ Universidad de San Buenaventura \\ Grupo de investigación Aerotech \\ Rol de investigador: teórico \\ https://orcid.org/0000-0001-9448-4963 \\ $\bowtie$ rsalazar@usbbog.edu.co
}

\section{Wilson Pinzón Velasco}

Magíster en en Ciencias

de la Ingeniería Química

Universidad de San Buenaventura

Grupo de investigación Aerotech

Rol de investigador: teórico

https://orcid.org/0000-0001-9553-3579

$\bowtie$ wpinzon@usbbog.edu.co

\footnotetext{
Artículo de investigación derivado del proyecto de investigación "Desarrollo de criterios normativos y protocolos de seguridad para lanzamiento y experimentación de vehículos tipo cohete dentro del territorio colombiano". Adscrito al Grupo de investigación Aerotech (coL0033088). Financiado por la Universidad de San Buenaventura, sede Bogotá.
}

Cómo citar este artículo: Bonilla Romero, J. S., Urrego Peña, J. A., Salazar, R., \& Pinzón Velasco, W. (2020). Desarrollo de criterios normativos y protocolos de seguridad para experimentación con cohetería dentro del territorio colombiano. Revista Ciencia y Poder Aéreo, 15(2), 77-93. https://doi.org/10.18667/cienciaypoderaereo.651 


Desarrollo de criterios
normativos y protocolos
de seguridad para
experimentación con cohetería
dentro del territorio colombiano

Development of Regulatory Criteria and Security Protocols for Rocketry Experimentation within Colombian Territory
Resumen: la evolución que ha tenido la industria aeroespacial a nivel mundial ha motivado a Colombia al desarrollo propio de tecnologías, normativas e investigaciones en este campo, de esta motivación nació este proyecto de investigación, el cual está dividido en dos fases. En el presente documento se expone la primera fase y lo que se espera obtener de la segunda. En primera instancia, se habla de la metodología que se adoptó para la culminación efectiva de su primera fase y la que se trabajará en el desarrollo de la segunda; luego se presenta el marco normativo, en el que se sustenta la investigación de entidades reguladoras internacionales, el cual puede tomarse como base, para la aplicación nacional; seguido a esto, están los antecedentes de esta investigación, como la historia de la cohetería en Colombia, desde los primeros lanzamientos experimentales, realizados hace sesenta años, el desarrollo de algunos protocolos experimentales expuestos en proyectos por algunas universidades y entidades, y lo realizado desde el 2013 por el semillero de investigación GIAS, de la Universidad de San Buenaventura, sede Bogotá, hasta el enlace con este proyecto. Por último, se presentan los resultados obtenidos durante la primera fase del proyecto y los que se esperan conseguir con el desarrollo de la segunda.

Palabras clave: aeroespacial; ingeniería aeronáutica; normativas; protocolos de seguridad; vehículos tipo cohete.

Abstract: The aerospace industry has evolved dramatically over the years, motivating the Colombian aerospace sector to undertake its own efforts regarding research and development. Such motivation promoted the two-phase research project that will be addressed in this work. The first part of this paper explains the methodology that was adapted to effectively conclude the first phase of the project and thus give a start to the second phase. Then, the article introduces the normative framework that supports research projects by international regulatory entities that could be adapted to national initiatives. Afterward, prior research studies on this subject and their contribution will be addressed, spanning over the history of rocketry in Colombia since the first experimental launches carried out about sixty years ago and the development of experimental protocols by Colombian universities and other institutions -including the work by San Buenaventura University (Bogotá) research group GIAS-, to finally connect these experiences with the objectives of this study. The last section presents the results obtained after the first phase of the project and what is expected during the second phase.

Keywords: Aerospace; aeronautical engineering; regulations; safety protocols; rockettype vehicles.

Resumo: A evolução que tem tido a indústria aeroespacial no mundo inteiro levou a Colômbia ao próprio desenvolvimento de tecnologias, normativas e pesquisas nesse campo. A partir dessa motivação, nasceu este projeto de pesquisa dividido em duas fases expostas no seguinte documento, onde no começo expõe-se a metodologia adotada para a efetiva conclusão da primeira fase, a qual funcionará no desenvolvimento da segunda. Em seguida, é dada a abertura de um marco normativo no qual sustenta-se a pesquisa de entidades reguladoras internacionais, que podem ser tidas em conta como base para a aplicação nacional. Posteriormente, apresenta-se tudo o que precede esta pesquisa, como a história da ciência dos foguetes na Colômbia, desde os primeiros lançamentos experimentais há sessenta anos até o desenvolvimento de protocolos experimentais expostos em projetos de universidades e entidades. Finalmente, apresenta-se o trabalho que foi feito desde 2013 pelo grupo de pesquisa GIAS da Universidade de San Buenaventura de Bogotá, até o vínculo com este projeto. Na parte final do trabalho são apresentados os resultados obtidos na primeira fase do projeto e os que esperam obter-se com o desenvolvimento da segunda.

Palavras-chave: Aeroespacial; Engenharia aeronáutica; Normativas; Protocolos de segurança; Veículos do tipo foguete. 


\section{Introducción}

La humanidad siempre ha soñado con volar más alto y llegar más lejos, y Colombia no se ha quedado fuera de esto; desde la década de los 60 también ha sido partícipe de lanzamientos experimentales en todo su territorio, con muchos éxitos y, claramente, con muchos fracasos, que han llevado a diferentes problemáticas y prohibiciones, pero aun así, siempre avanzando, aprendiendo y desarrollando mejores estructuras, sistemas y procedimientos, basándose en la experiencia y en la teoría internacional. Actualmente, a nivel nacional no hay normativas y protocolos de seguridad establecidos, específicamente para el lanzamiento y experimentación con vehículos tipo cohete, y de aquí surge la necesidad de crear un proyecto de investigación, que tenga como fin crear protocolos de seguridad, basados en normativas internacionales adaptadas al contexto nacional, en los que se plasme el procedimiento logístico previo, durante y posterior a los lanzamientos de este tipo, para así, desarrollar su posible implementación.

El presente documento plasma el objetivo principal de esta investigación, el cual está centrado en el desarrollo y la aplicación de normas y protocolos de seguridad para el lanzamiento y experimentación de vehículos tipo cohete, dentro del territorio colombiano. Para lograrlo, se verifican los antecedentes, observando la historia de la cohetería en Colombia, los protocolos de seguridad utilizados en lanzamientos experimentales en los últimos años y el impacto que ha tenido el semillero de investigación de la Universidad de San Buenaventura, sede Bogotá, GIAS, perteneciente al grupo de investigación Aerotech. Se toma como marco teórico de referencia, las normativas de cohetería utilizadas en los Estados Unidos y en el Reino Unido, países en los que estos procedimientos están completamente informados y normalizados, para su segura experimentación. Por último, se llega a los resultados obtenidos en la primera fase de este proyecto, los cuales son las matrices de caracterización de normativas de origen americano e inglés, las matrices de riesgos implicados al momento de la experimentación con vehículos tipo cohete y, se expone el planteamiento de los primeros criterios normativos aplicados a este tipo de experimentación. Dicho planteamiento da apertura a la segunda fase del proyecto, de la cual se esperan obtener principalmente dos productos, primero, la caracterización y adaptación de las normativas obtenidas en el primer producto de la fase uno, y segundo, desarrollar los protocolos de seguridad, junto con las listas de chequeo y la verificación de todo el proceso, para la experimentación con vehículos tipo cohete dentro del territorio colombiano.

\section{Metodología}

Esta investigación basa sus principios metodológicos en la experimentación científica y su posterior observación, con lo cual se puede analizar la conexión entre la causa y el efecto de ciertas situaciones. Este proyecto fue dividido inicialmente en dos fases claves para su desarrollo, la primera estuvo enfocada en el establecimiento inicial de criterios normativos preliminares, que rigieran el procedimiento de lanzamiento y experimentación de vehículos tipo cohete, dentro del territorio nacional; $y$ la segunda fase, consiste en el desarrollo y la aplicación de tales criterios normativos y protocolos de seguridad, para este tipo de eventos dentro del territorio colombiano.

Así mismo, la primera fase se dividió en cuatro etapas primordiales. La primera etapa estuvo encaminada a la búsqueda, adquisición y lectura de diferentes normativas internacionales, utilizadas para la regulación de investigación y lanzamientos de cohetería experimental, teniendo en cuenta normativas de origen americano, canadiense e inglés. La segunda etapa consistió en la recolección de información, referente a las necesidades y requerimientos básicos para la experimentación y el lanzamiento de cohetería a nivel nacional, esto con ayuda de diferentes fuentes, como mesas de discusión y entrevistas a personas con el conocimiento pertinente que aportaron a la investigación. 
En la tercera etapa se construyó una matriz de riesgos con la información referente a los riesgos presentes durante el alistamiento, lanzamiento, embarque, traslado y desembarque de vehículos tipo cohete dentro del territorio colombiano, clasificada por los siguientes factores: (a) personal expuesto durante el proceso, (b) peligros que pueden existir, (c) posibles efectos de este, (d) controles existentes, (e) evaluación del riesgo, (f) valoración del riesgo, (g) criterios para establecer funciones y (h) las medidas de intervención para la mitigación del peligro. Adicionalmente, se sintetizó la información sobre los tipos de peligro presentes como son: biológicos, físicos, químicos, psicosociales, biomecánicos, por condiciones de seguridad o por fenómenos naturales, además de tener en cuenta algunos incidentes asociados a la seguridad, según la Norma ANSI z 16.2, asociados a la higiene personal y acciones causantes de peligro. De igual forma, se establecieron criterios normativos, que permiten la comparación de diferentes aspectos a evaluar entre las normativas NAR, Trípoli, UKRA, NFPA y FAA, aspectos como: (a) materiales del cohete, (b)sistema de encendido, (c) lanzadera, (d) tamaño, (e) seguridad de vuelo, (f) sistema de recuperación, (g) requisitos de certificación, (h) ubicación del lanzador (piloto o personal asistente), (i) distribución y estructura del documento reglamentario, (j) alternativa o soporte con otra normativa, (k) procesos de aseguramiento del documento, (l) caracterización de participación o acceso, $(\mathrm{m})$ determinación de la máxima autoridad en el documento, ( $\mathrm{n}$ ) materiales de construcción de los motores, (o) condiciones de uso de estos, ( $p$ ) condiciones restrictivas según estas normativas, (q) protocolos de seguridad o actividades procedimentales, (r) el año de publicación, aceptación o vigencia, y (s) la referencia donde se puede encontrar la información.

Para finalizar, como cuarta etapa, se planteó el desarrollo de la fase dos del proyecto, estableciendo preliminarmente los criterios normativos y los protocolos reglamentarios de seguridad, para el lanzamiento de cohetería tanto atmosférica, como estratosférica dentro del territorio. De igual modo, se propone realizar el lanzamiento de un cohete atmosférico funcional, con el fin de corroborar la aplicabilidad del cumplimiento de la normativa y los procedimientos propuestos en este proyecto.

La segunda fase, que se encuentra en proceso, está dividida en tres etapas fundamentales, que a su vez, se subdividen en algunas tareas específicas para su culminación efectiva. La primera etapa del proceso consiste en la elaboración de los protocolos de seguridad, de las listas de chequeo, y su verificación, en principio se investigan las bases en las que estará sustentada esta fase del proyecto, como la consulta de los antecedentes históricos sobre la experimentación de cohetería a nivel nacional, los antecedentes protocolarios de algunos entes educativos y los antecedentes experimentales del semillero de investigación GIAS, también se caracterizan las normativas de cohetería utilizadas en otros países, como en los Estados Unidos y el Reino Unido. Con base en lo anterior, se realizará el documento referente a los protocolos de seguridad, junto con las listas de chequeo y verificación respectivas, para llevar a cabo un procedimiento efectivo al momento de realizar un lanzamiento o la experimentación con este tipo de vehículos dentro del territorio colombiano.

En la segunda etapa, se llevará a cabo la gestión y la elaboración del lanzamiento del cohete USB-Rocket I y sus réplicas, para la comprobación de la eficiencia de los protocolos de seguridad; esta etapa está subdividida en dos partes, una consiste en la adquisición de equipos especializados para el cohete, y la otra consiste en la gestión de los permisos con las entidades reguladoras pertinentes, para realizar el lanzamiento y, de esta forma, pasar a la última etapa del proceso.

En suma, la tercera etapa está enfocada en el análisis del desempeño de los criterios normativos adaptados y protocolos de seguridad, junto con sus listas de chequeo y verificación, en caso de conseguir realizar la prueba experimental de estos durante los lanzamientos. Posterior a esto, se realizan las correcciones pertinentes, basadas en la observación durante las pruebas y, por último, se elabora la documentación respectiva, en la cual se plasman los resultados comprobados 
obtenidos en el proyecto, para su futura implementación a nivel nacional.

\section{Normativa base}

Se tienen como eje principal, las normativas utilizadas en Estados Unidos y el Reino Unido, donde en el transcurso de los años, han conformado una serie de parámetros para tener en cuenta al momento de experimentar o lanzar vehículos tipo cohete, dentro del territorio nacional. Los parámetros más importantes, informados en la gran mayoría de las normativas, contienen información primordial como: (a) materiales del cohete y del motor, (b) sistemas de encendido, (c) fallos al momento del lanzamiento, (d) forma de la lanzadera, (e) tamaño del cohete, (f) seguridad de vuelo, seguridad en la recuperación, (g) localización del piloto o del personal asistente al lanzamiento, (h) condiciones de uso de motores comerciales o experimentales, (i) restricciones, (j) protocolos de seguridad y (k) documentación requerida para realizar estos procedimientos, información de alta relevancia pero de escasa existencia dentro del territorio colombiano. Las normativas tomadas para la realización de este proyecto se enumeran a continuación.

\section{NAR (National Association of Rocketry)}

Esta es la asociación deportiva de cohetería más antigua y grande del mundo. Fundada en 1957 en los Estados Unidos (NAR, 2012b), ha contado con la unión de una gran variedad de clubes, lo que les ha permitido avanzar, a la vez, en regulación y control de los lanzamientos de sus cohetes. El código de seguridad utilizado por la NAR está dividido en tres segmentos importantes; inicialmente, todo lo que sea cohetería a escala, realizada por grupos juveniles para su experimentación (NAR, 2012c); en segundo lugar, lo referente al programa de entrenamiento para oficiales de seguridad, encargados del control al momento de la experimentación (NAR, 2000); y el tercer segmento está dirigido a los aficionados, que quieren ir más allá de solo la cohetería a escala y deciden construir cohetes de alta potencia (NAR, 2012a).

\section{TRA (Tripoli Rocketry Association)}

Esta es una organización sin fines lucrativos, con propósitos de avance tecnológico y operación de cohetes de alta potencia creada a finales de 1964 en los Estados Unidos, junto con 22 países asociados de todo el mundo (TRA, 2004). A diferencia de la NAR, antes mencionada, esta organización fomenta el crecimiento científico de la experimentación con vehículos tipo cohete de alta potencia. La normativa utilizada en esta organización tiene siete divisiones, la primera corresponde al código de seguridad, en el cual se definen las reglas y políticas exclusivas para lanzamientos de investigación de Trípoli (TRA, 2017b); en la segunda división se encuentra todo lo referente a la seguridad para el uso de cohetes de alta potencia (TRA, 2017a); la tercera es un documento guía para el personal interesado en el puesto de oficial de seguridad, en el rango de experimentación, para promover la práctica sana y segura de estos procedimientos (TRA, 2015b); en la cuarta división está la recomendación de uso de materiales, especialmente metales, que se pueden utilizar para la cohetería de alta potencia (Kelly, 2015); la quinta división, generada por esta organización, consiste en una variedad de consejos (no reglas) dados a sus integrantes, para llevar a cabo los procesos de experimentación con seguridad (TRA, 2015a); en la sexta, realizada por la unión entre TRA, NAR, S\&T (Standards and Testing Committee) y CAR (Canadian Association of Rocketry), se muestra un listado de los motores certificados por las organizaciones para el uso en cohetería (NAR S\&T, 2020); y para cerrar, en la séptima división, se encuentra una guía resumida de los códigos y políticas de seguridad de los lanzamientos (TRA, 2017C).

\section{UKRA (United Kingdom Rocketry Association)}

Esta asociación representa los cohetes de alta potencia, potencia media, modelos y cohetes realizados por aficionados o de carácter experimental, en el Reino Unido, desde 1996 (UKRA, 2006). UKRA cuenta con una certificación que demuestra la competencia en operación por parte de sus integrantes, al momento de la manipulación de diferentes tipos de motores de 
cohetes y diferentes niveles de potencia, esta certificación está dividida en dos categorías, la categoría para mayores (mayores de 18 años) que está subdividida en 3 niveles, los cuales dependen del tipo de motor que se quiera lanzar dentro del cohete. El primer nivel es para el lanzamiento de cohetes de impulso tipo $\mathrm{H}$ o I, según el seguro BMFA (British Model Flying Association), el segundo nivel corresponde a motores de impulso tipo J, K O L, según el seguro UKRA, y el tercer nivel, al lanzamiento de motores de impulso tipo $\mathrm{m}$ u o, según el seguro UKRA. De igual manera, cuenta con un cuarto nivel que corresponde a los oficiales de seguridad o RSo, encargados del control durante los lanzamientos o eventos que correspondan al nivel para el cual están certificados (UKRA, 2015). Y la categoría para menores, certificación junior (de 12 a 18 años de edad), está subdividida en dos niveles con la misma restricción de tipo de impulso, que tienen los dos primeros niveles de la categoría para mayores (UKRA, 2018). Para concluir, también cuenta con la guía de estudio para la preparación de los exámenes de cada uno de los niveles anteriormente enunciados y los formularios necesarios para estos procesos (UKRA, 2017).

\section{NFPA (National Fire Protection Association)}

Desde 1896 en los Estados Unidos, esta asociación sin fines de lucro mantiene los estándares de seguridad para la prevención de lesiones, pérdidas económicas o la misma muerte, causadas por incendios, riesgos eléctricos o accidentes relacionados con estos (NFPA, 2019). La asociación tiene un completo conjunto de normas, con más de 300 códigos, de los cuales, para cohetería los más importantes son tres: (a) NFPA 1122: código para cohetes modelo, en el cual se enuncia toda la parte de diseño, construcción, limitaciones y confiabilidad, que deben tener en cuenta los aficionados al momento de realizar estos modelos (NFPA, 2018b); (b) NFPA 1125: estándares para comunicación de servicios de emergencia, los cuales cuentan con un plan de consolidación, aprobado por esta asociación para el uso en caso de cualquier tipo de emergencia (NFPA, 2020); y (c) NFPA 1127: código para cohetes de alta potencia, en el cual se plantean los requisitos importantes a la hora de manipular cohetes de esta gama, para evitar lesiones del usuario y del público asistente (NFPA, 2018a).

\section{FAA (Federal Aviation Administration)}

Recibe este nombre a partir de 1967, pero desde muchos años atrás, esta asociación ya controlaba cada elemento que se mueve a cierta altura del suelo, con diferentes regulaciones desde la creación de la industria aérea en 1925 (FAA, 2017). Para cohetería, en el capítulo 14, parte 101, sub-parte c, se encuentra todo lo correspondiente a cohetería experimental, limitaciones de operación, tanto para modelos como para cohetes de alta potencia y cohetes avanzados, y la información requerida para los lanzamientos y sus requerimientos (FAA, 2009).

\section{Antecedentes}

\section{Antecedentes históricos colombianos}

Apasionados por el lanzamiento del Sputnik 1, el primer satélite artificial puesto en órbita por parte de la Unión Soviética, el 4 de octubre de 1957 (BBC, 2017), algunos intrépidos colombianos comenzaron a experimentar, a inicios de los 60, en esta carrera espacial. Uno de los pioneros fue Francisco Restrepo Gallego, quien alcanzó a realizar el lanzamiento de una gran cantidad de cohetes, de forma experimental, desde 1960 (Aeropuerto de Medellín Olaya Herrera, 2018), tuvo éxito en muchos lanzamientos, y a su vez, algunos imprevistos, los cuales le hicieron retirarse por seguridad de este campo, pero aún en su retiro, con mucha pasión desde ese entonces, se ha empeñado y destacado en la construcción de modelos a escala de aeronaves, cohetes y naves espaciales, las cuales ha donado a diferentes instituciones, principalmente en la ciudad de Medellín.

Simultáneamente, con el ingeniero Restrepo, el tocaimuno Isaías Moreno Moncada fascinado con el primer elemento lanzado hacia el espacio, comenzó a experimentar con sus propios prototipos, como el Seek 1 , el cual no despegó del suelo, pero se desplazó paralelo a este; el Seek 2, que alcanzó a elevarse 80 metros; 
el Tequendama 1, que se elevó a los 300 metros de altura; el Vilvar 1 , que contenía un cuy en su interior, pero explotó metros después de su despegue; y por último, uno de los más representativos en esta época para él, el Tequendama 2, el cual consiguió llegar a una altura de 380 metros y descender exitosamente con un paracaídas de ayuda, conteniendo dentro un primate apodado por los medios de comunicación el "micronauta" (Pirateque-Bolívar \& Sabogal, 2011).

Así mismo, junto a estos pioneros se encuentran más colombianos que han experimentado y explorado el mundo aeroespacial, como Hernán Charry, el caqueteño que tuvo ciertos problemas con las autoridades en su juventud, por haber utilizado erradamente una fórmula química en sus primeros cohetes y haber hecho que explotaran en zonas públicas y residenciales, hecho que solucionaría en los años 90 , con el lanzamiento de varios cohetes a menos de un kilómetro de altura (Redacción el tiempo, 1998); y Alberto Quijano Vodniza, un pastuso que a sus once años de edad intentó comenzar en esta carrera espacial con un cohete a base de pólvora, que quemó la ropa de sus padres, quienes le impusieron una prohibición a sus experimentos (Redacción el tiempo, 2007).

Estas pequeñas hazañas provocaron a nivel nacional un gran deseo de experimentación y aprendizaje por parte de la población colombiana, creando así, en 1988, el Programa Aeroespacial Colombiano (P.A.C) y más adelante, en 1998, un proyecto concreto por iniciativa del científico Jorge Reynolds, llamado Trabajo Aeroespacial de Misión Satelital TAMSA; y con ayuda de los ingenieros Edgard Espejo y Wilson Pinzón, incentivaron la investigación en algunas instituciones, como INDUMIL, el SENA, la Universidad de San Buenaventura y la Universidad Nacional de Colombia, entre otras, las cuales contribuyeron bastante en el avance teórico-práctico de este tema. Con el ánimo de organizarse de una mejor forma, se creó la Asociación Pro Agencia espacial ASPA, que produjo un mayor movimiento, tanto en los individuos, como en las organizaciones. Se creó el Nodo de estudios aeroespaciales, Napsú, en la Universidad Nacional de Colombia; INGES AEROSPACE, en la Universidad de Antioquia; Pléyades, en la Universidad Distrital Francisco José de Caldas; la Asociación de Astrónomos Autodidactas en Colombia, Asasac; la Comisión Colombiana de Cohetería y Astronáutica, C3; y la Comisión Colombiana del Espacio, CCE; esta última reposando en la cabeza de la Fuerza Aérea Colombiana desde el 2012, donde se desarrollan los primeros proyectos para la creación de nanosatélites colombianos de observación (Urbina-Carrero, 2017), de los cuales cabe resaltar el lanzamiento del FACSAT-1, el satélite de observación de la Fuerza Aérea Colombiana fabricado por la empresa danesa Gomspace, efectuado el 28 de noviembre del 2018, en el multilanzamiento de la Agencia Espacial de la India (IRso) (León-Lozada, 2018). De igual manera, se han constituido otras entidades, aquí no enunciadas, de carácter gubernamental y algunas de iniciativas privadas, que han permitido el desarrollo e impulso de esta industria a nivel nacional hasta la actualidad.

Llegando al año en curso (2020), el Consejo Nacional de Política Económica y Social, CONPES, formula una Política de Desarrollo Espacial, el CONPES 3983, que promueve a las tecnologías espaciales que impulsen la productividad, diversificación y sofisticación de la economía colombiana (CONPES, 2020). El trayecto de esta política se divide en tres secciones, inicia con la declaración de uso tecnológico, la cual indica que no es utilizada únicamente en el campo militar, también los inversionistas y las empresas privadas encuentran un campo amplio, para el desarrollo de sus actividades en la industria espacial, y sigue así hasta que el acceso al espacio ya no es restringido para un cerrado número de países. En congruencia con lo anterior, desde finales de los años 70 se aprobaron tres documentos CONPES, los cuales tenían como finalidad la compra de tres satélites, dos de comunicaciones y uno de observación de la Tierra, desafortunadamente para el país no se obtuvieron, solo quedaron consignados en los CONPES 1421, 3579 y 3683 respectivamente, debido a que no se tenía una estrategia clara para la explotación de los beneficios de este tipo de tecnologías y así impulsar el desarrollo del país en este ámbito. El CONPES 3983, realizado este año durante el mes de enero, trata tres ejes fundamentales para su finalización a largo plazo; en primera instancia, propone la generación de estrategias teniendo en cuenta el 
potencial, las oportunidades y capacidades existentes actualmente a nivel nacional; en segundo lugar, se propone una hoja de ruta para identificar las barreras que presentan las iniciativas privadas, las dificultades para acceder a estos mecanismos y cómo superar estas eventualidades; y en tercer lugar, plantea la búsqueda de la definición de un entorno institucional, acorde con las necesidades y la realidad de este sector, buscando acciones de mejora en diferentes ámbitos para el desarrollo en materia satelital y espacial a nivel nacional. Finalmente, se establece que esta política tiene un costo total estimado de 1.904 millones de pesos, para cumplir a cabalidad los objetivos en este planteamiento (CONPES, 2020).

\section{Antecedentes protocolarios}

A lo largo de la historia contada, sobre el desarrollo experimental de la cohetería en Colombia, se ha prestado poca atención a los protocolos de seguridad de logística, previos, durante y posteriores a los lanzamientos y experimentación con vehículos tipo cohete, principalmente en lanzamientos realizados en el siglo pasado (siglo $\mathrm{xx}$ ), por eso se han tenido muchos inconvenientes y se han presentado muchas prohibiciones por parte de las autoridades competentes, por posible afectación a la seguridad pública. Pero esto ha cambiado en el siglo actual, debido a que se han tenido como base protocolos internacionales de seguridad y factores empíricos, para realizar algunos procedimientos y listas de chequeo, buscando evitar la mayor cantidad de riesgos posibles y poder continuar con la experimentación y el avance científico de este campo de forma segura.

Comenzando en el 2008, la Agencia Espacial Japonesa, JAXA, con ayuda del profesor Nobuaki Ishii conforma el manual titulado "Cohetes de agua, Manual del educador", el cual fue traducido al español por la Organización de las Naciones Unidas para la Educación, la Ciencia y la Cultura, unesco. Este manual es el instructivo para la elaboración de cohetes a base de agua con materiales de fácil adquisición, fue diseñado para que los maestros infundieran en los jóvenes la curiosidad científica, con el entendimiento básico de los cohetes de este tipo. El manual está estructurado de una forma muy completa para este tipo de cohetes. Inicialmente describe los preparativos necesarios para la construcción; luego el procedimiento para su fabricación y la fabricación de su lanzador; el protocolo necesario para el momento del lanzamiento del cohete, teniendo en cuenta la seguridad que este procedimiento requiere; $y$ finalmente, una explicación de los principios que rigen los vuelos de estos cohetes (Organismo de Exploración Aeroespacial del Japón, JAXA, 2008).

Siguiendo así, en el 2009, el entonces ingeniero en formación José Alejandro Urrego Peña de la Universidad de los Andes, lanzó el cohete con combustible sólido AINKAA I - Misión Séneca I, el cual alcanzó una altura de vuelo de 780 metros (Urrego, 2009), una gran altura, aunque presentó una falla en el despliegue del paracaídas al momento de su retorno a tierra, provocando que se incrustara aproximadamente a un metro de profundidad en el suelo. En este proyecto se realizó un protocolo de seguridad, basado en el código de seguridad para experimentación amateur de la Asociación de Cohetería Experimental y Modelista Argentina, ACEMA (ACEMA, 2007), aunque sin abarcar todo el documento de la Asociación, se realizaron unas listas de chequeo, las cuales establecen parámetros para tener en cuenta al momento del ensamble del cohete, de la inspección del inventario, del montaje del cohete, de la ignición de este y de finalización de la misión, cuando el cohete regresa a tierra; además, plantea algunas observaciones de tipo logístico y procedimental, que deberían tenerse en cuenta durante el proceso. El seguimiento de este protocolo permitió que la misión se llevara a cabo de forma segura, ordenada y con solo un imprevisto al momento del retorno del elemento a tierra, por condiciones atmosféricas diferentes a las tenidas en cuenta al momento de la simulación (Urrego \& Rojas, 2010).

En el 2010 los entonces ingenieros en formación, Felipe Alejandro Riveros y Luis Alejandro Rodríguez, de la Universidad Militar Nueva Granada, diseñaron y construyeron el cohete llamado LARFAR I, el cual fue lanzado en la base militar de Tolemaida (Riveros-Enciso \& Rodríguez-Herrera, 2010), pero antes de su lanzamiento 
realizaron la prueba del motor en las instalaciones de INDUMIL, para la cual siguieron un protocolo de seguridad basado tanto en el código de seguridad para la práctica de cohetería experimental amateur, de la Asociación de Cohetería Experimental y Modelista del Uruguay (ACEMU, 2008), como en el código de la ACEMA. Este protocolo estaba conformado por los parámetros a tener en cuenta del banco de pruebas, las dimensiones y el aspecto físico del campo donde será realizada la prueba; los elementos de seguridad necesarios, los factores a considerar durante el transporte y el ensamble del motor; la información general de la prueba; y finalmente, el procedimiento paso a paso que se debe llevar a cabo de manera previa, durante y posterior a la ignición del motor. Este procedimiento utilizado en la prueba del motor permitió tomar los datos de forma segura, ordenada y adecuada, además de observar el comportamiento que tendría la tobera ante perturbaciones externas, datos importantes para el posterior lanzamiento del cohete.

Posterior a esto, en el 2012, a cargo del entonces Maestro en formación José Alejandro Urrego Peña, de la Universidad de los Andes, se llevó a cabo el estudio del alcance de un cohete con propulsión líquida hasta una altura estratosférica de $11 \mathrm{Km}$ (Urrego, 2012), continuando así, con la misión Séneca Il y con la construcción del cohete AINKAA II. Para esta misión se planteó un procedimiento de seguridad, basado en la misión anterior y teniendo en cuenta consideraciones de manipulación y almacenamiento de los elementos; las hojas de seguridad (MSDS) correspondientes al oxígeno líquido, nitrógeno gaseoso y gasolina común, donde se encuentra la información de posibles peligros, y del correcto manejo y almacenamiento; las listas de chequeo de todo el procedimiento para el lanzamiento teniendo en cuenta diferentes circunstancias, como la no ignición del motor o la explosión de este y la especificación del procedimiento de seguridad para el llenado de los tanques de combustible y oxidante del motor, y los riesgos asociados a este proceder. Este protocolo permitió llevar a cabo la experimentación de forma segura, como el procedimiento de seguridad contemplaba el caso de no ignición del motor, que fue lo sucedido en las pruebas preliminares por causa de un mal funcionamiento de las válvulas de control de fluidos, se supo cómo proceder para culminar la misión sin otro imprevisto o accidente (Urrego, 2012).

En el 2015, el Grupo de Investigación y Desarrollo Aeroespacial de la Universidad Nacional de Colombia, GIDA - UN, realizó la construcción y el planteamiento del lanzamiento del SIMTE II, en la misión Prometeo I, a una altura de entre 2,5 y 3 kilómetros (Álvarez et al., 2015). Para esta misión se basaron en la metodología utilizada en el Space Mission Engineering: The new SMAD, para establecer algunos parámetros de seguridad en todo el lanzamiento, lo dispuesto por parte del grupo de investigación establece toda la operación en tierra dividida en el equipo de lanzamiento, la seguridad en el campo de lanzamiento, la gestión de personal referente a su función dentro de la misión, la infraestructura de la operación en tierra, los protocolos para cada una de las etapas de la misión, teniendo en cuenta emergencias y diferentes situaciones, y el transporte respecto a los embalajes y las recomendaciones pertinentes. Estos procedimientos hasta el día de hoy no han sido probados, debido a que no se ha puesto en marcha la misión Prometeo I, la metodología también otorga parámetros que deben tenerse en cuenta a la hora de realizar estos procedimientos, como la gestión pertinente del personal encargado dentro de la misión (Álvarez et al., 2015).

Finalizando, en el 2018 los entonces ingenieros en formación Juan Sebastián Serrato y Daniela Ramírez, de la Universidad de San Buenaventura en conjunto con la Universidad de los Andes, y con apoyo de la Asociación de Egresados de la Universidad de los Andes Uniandinos y la Fuerza Aérea Colombiana, realizan el potencial lanzamiento del cohete AINKAA Alpha-Misión Séneca $v$ de alcance estratosférico. Para este proyecto se realizaron protocolos basados en los estándares de seguridad de la National Aeronautics and Space Administration (NASA) y en protocolos utilizados en las misiones anteriores de la Universidad de los Andes. También tuvieron la posibilidad de asistir al lanzamiento del cohete AINKAA V - Misión Séneca VII, realizado en la base aérea del GAORI (Fuerza Aérea Colombiana), en Marandúa, Vichada, dado que actualmente se realizan investigaciones en lanzamiento de cohetes y esta base 
ha sido utilizada por universidades para tales efectos (Murcia-Piñeros \& Portilla-Barbosa, 2014). Este lanzamiento permitió conformar los protocolos de seguridad más robustos expuestos hasta el momento en este trabajo, los cuales abarcan parámetros, como: (a) la capacitación del personal que está a cargo, (b) la asistencia de cada una de las personas involucradas, (c) la puesta a punto del cohete y de la zona de lanzamiento, (d) los procedimientos secuenciales de disparo del vehículo, (e) la prevención y atención de cada uno de los riesgos presentes durante el proceso, (f) la secuencia cronológica de las actividades programadas, (g) los datos atmosféricos del campo en el momento del lanzamiento, (h) los procedimientos en caso de imprevistos o accidentes, e (i) procedimientos a seguir una vez culminada la misión (Serrato \& Ramírez, 2018). Este ha sido uno de los protocolos que más aspectos abarca, debido a su amplia variedad de parámetros relevantes a tener en cuenta para velar por la seguridad y eficiencia de la misión, hasta la detención de esta por un inconveniente con los ignitores que no permitió culminar todo el lanzamiento.

\section{Antecedentes del Semillero de Investigación en Asuntos Espaciales GIAS}

El semillero de investigación GIAS pertenece al grupo de investigación Aerotech, del Programa de Ingeniería Aeronáutica de la Universidad de San Buenaventura, sede Bogotá. Desde sus inicios se ha inclinado hacia la creación, aprobación y apropiación de criterios normativos y procedimentales, para el desarrollo de la investigación en el ámbito aeroespacial en Colombia; han realizado diversas actividades desde su reactivación en el 2013, año en el que reiniciaron sus actividades de desarrollo científico e investigativo. Para el período comprendido entre los años 2014 y 2016, realizaron actividades de fomento y motivación dirigidas al público infantil, propiciando su inmersión en el mundo de la cohetería educativa, como herramienta de proyección social, asistiendo a diferentes charlas y actividades en las que incentivaban, divulgaban y enseñaban conceptos sobre la construcción y aplicación de este tipo de elementos. Una de sus múltiples memorias puede observase en la figura 1 , llevada

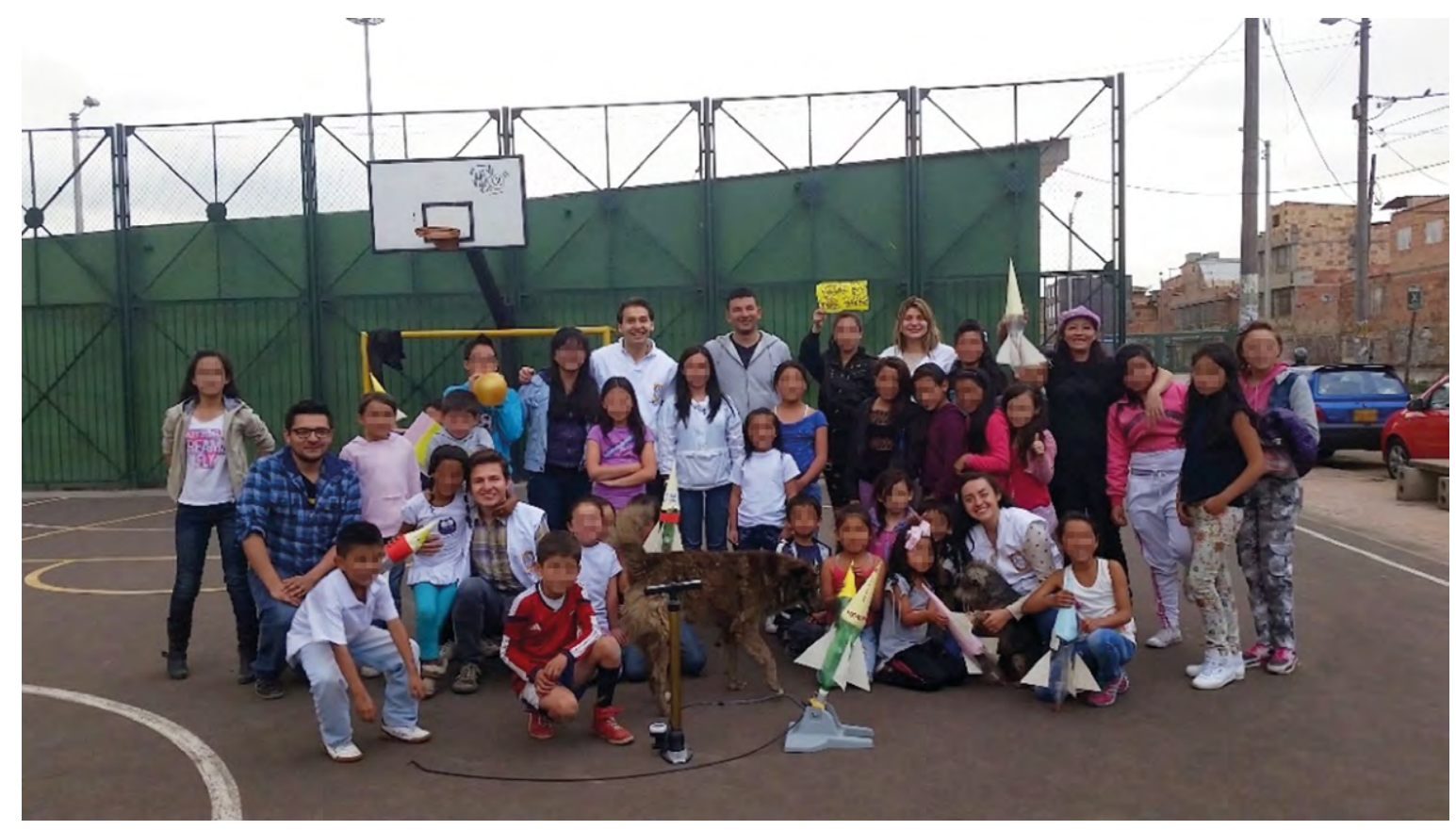

Figura 1. Actividad de divulgación en tecnología aeroespacial. Barrio Villa de los Comuneros Fuente: los autores. 
a cabo en octubre del 2014, en el barrio Villa de los comuneros, en el sur de Bogotá.

Explorando las diferentes oportunidades de desarrollo tecnológico a las que el semillero GIAS tiene acceso para aportar en la construcción de un planteamiento normativo, halló en las tecnologías de manufactura aditiva una solución viable para el desarrollo de prototipos de cohetes funcionales. En el 2016 se iniciaron procesos de manufactura de elementos fabricados con el método de modelado por deposición fundida o FDM, por sus siglas en inglés, permitiendo avanzar en procesos de manufactura de diferentes elementos aeronáuticos y aeroespaciales, como el prototipo de cohete usB-Rocket Réplica I que se observa en la figura 2.

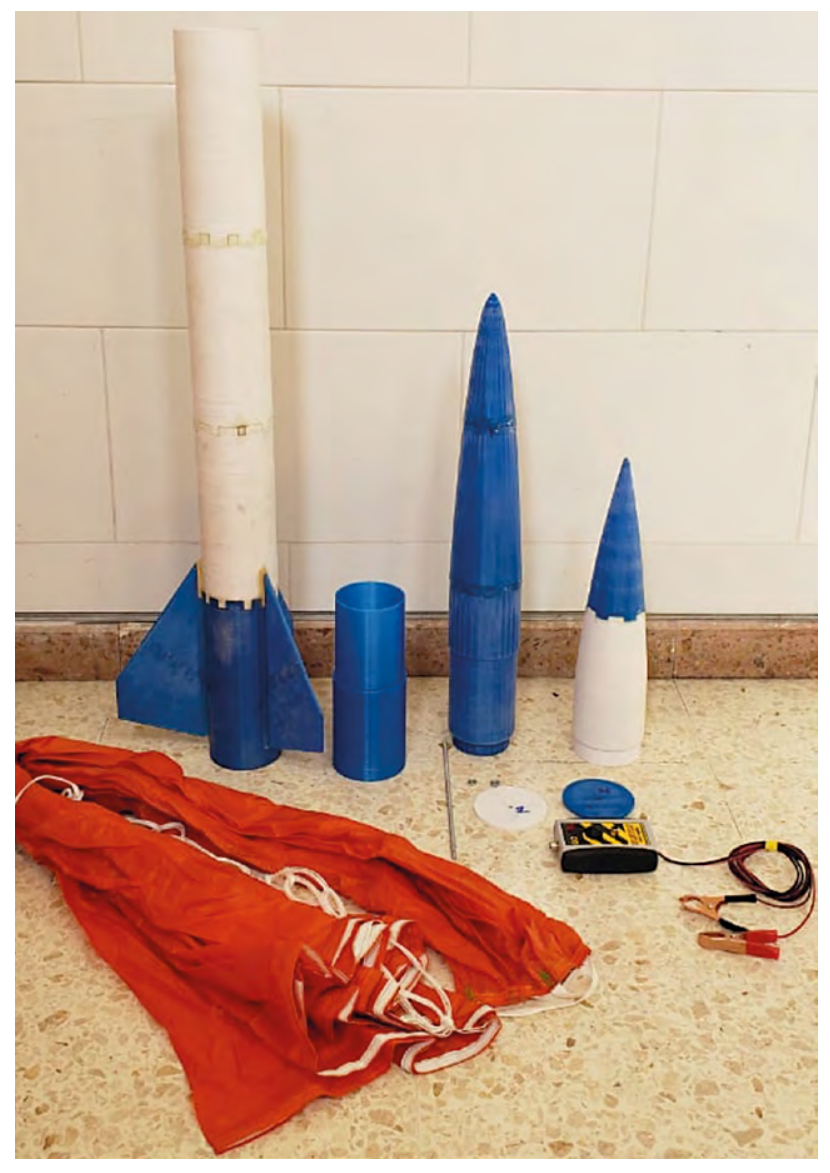

Figura 2. Cohete USB-Rocket Réplica I realizado enteramente con manufactura tipo FDM Fuente: los autores.
Con procesos de investigación como el mencionado, el semillero ha conseguido realizar diferentes alianzas estratégicas que le han permitido tanto el avance científico, como el desarrollo de diferentes investigaciones y el crecimiento como ente formador de la investigación académica; estas alianzas se han realizado con entidades e instituciones a nivel nacional, como la Fuerza Aérea Colombiana, la Universidad de los Andes y Uniandinos, entre otros; y a nivel internacional, con la TRA y la Asociación de Cohetes Experimentales del Pacífico (AERO-PAC), que es la prefectura del norte de California y el norte de Nevada de la Trípoli. Desde el 2014, el semillero GIAS ha realizado diferentes misiones de investigación con los entes ya nombrados. En la figura 3 se observa uno de los lanzamientos realizados por los estudiantes del semillero, en conjunto con el Proyecto Uniandino Aeroespacial (PUA) del Departamento de Ingeniería Mecánica de la Universidad de los Andes. Este lanzamiento se realizó en agosto del 2014, en la ciudad de Villa de Leyva, Boyacá-Colombia. Allí se observan momentos previos a la reunión de alistamiento normativo y protocolario, para el lanzamiento de la misión de investigación biológica Séneca Ix, la cual tenía como objetivo el estudio de cambios de tipo genético en nematodos (Caenorhabditis Elegans), al ser expuestos a condiciones de aceleración excesivas (mayores a 10 g), proporcionadas por un cohete sonda experimental (PUA, 2014), utilizando procedimientos secuenciales desarrollados por los investigadores, basados en normativas internacionales y autorizados por la Fuerza Aérea Colombiana para poder realizar el lanzamiento.

Posteriormente, en julio del 2016, el semillero junto con la Universidad de los Andes y el Capítulo Zero $\mathrm{G}$ de la Asociación de Egresados de la Universidad de los Andes, participó en el lanzamiento del vehículo cohete AINKAA III, con un apogeo de vuelo estimado de 4 kilómetros de altura sobre el nivel de lanzamiento, con el fin de realizar un proyecto de divulgación internacional con la Universidad de Costa Rica (PUA, 2016b). Una memoria de este evento se observa en la figura 4, en uno de los campos de la Universidad de Costa Rica, en Liberia-Guanacaste. 
Más adelante, en diciembre también del 2016, el mismo grupo de investigadores y la Fuerza Aérea Colombiana, se propusieron llevar a cabo la Misión Séneca VII, con el objetivo de lanzar el prototipo AINKAA $\mathrm{v}$, con capacidad de alcanzar la estratósfera, el cual se observa en la figura 5 (PUA, 2016a). Además de los proyectos, el semillero ha realizado diferentes investigaciones con estas entidades, lo cual le ha permitido crecer en su investigación y, así mismo, aportar a otras universidades y al sector aeroespacial colombiano, en gran medida, al avance científico.
Como último proyecto cabe incluir, que en el 2018 con el cohete mostrado en la figura 6, perteneciente al Programa de Ingeniería Aeronáutica de la Universidad de San Buenaventura, sede Bogotá, se obtuvo el certificado en lanzamiento de cohetes de alta potencia Nivel II, expedido por la Tripoli Rocketry Association, en Nevada, Estados Unidos, donde se probaron y se experimentaron dos prototipos del cohete USB Rocket I y dos tipos de propulsores sólidos comerciales: motor-cohete Aerotech H195T-14A y Aerotech J270W-14A, con un impulso total de $236 \mathrm{~N}-\mathrm{S}$ y $703 \mathrm{~N}$-S

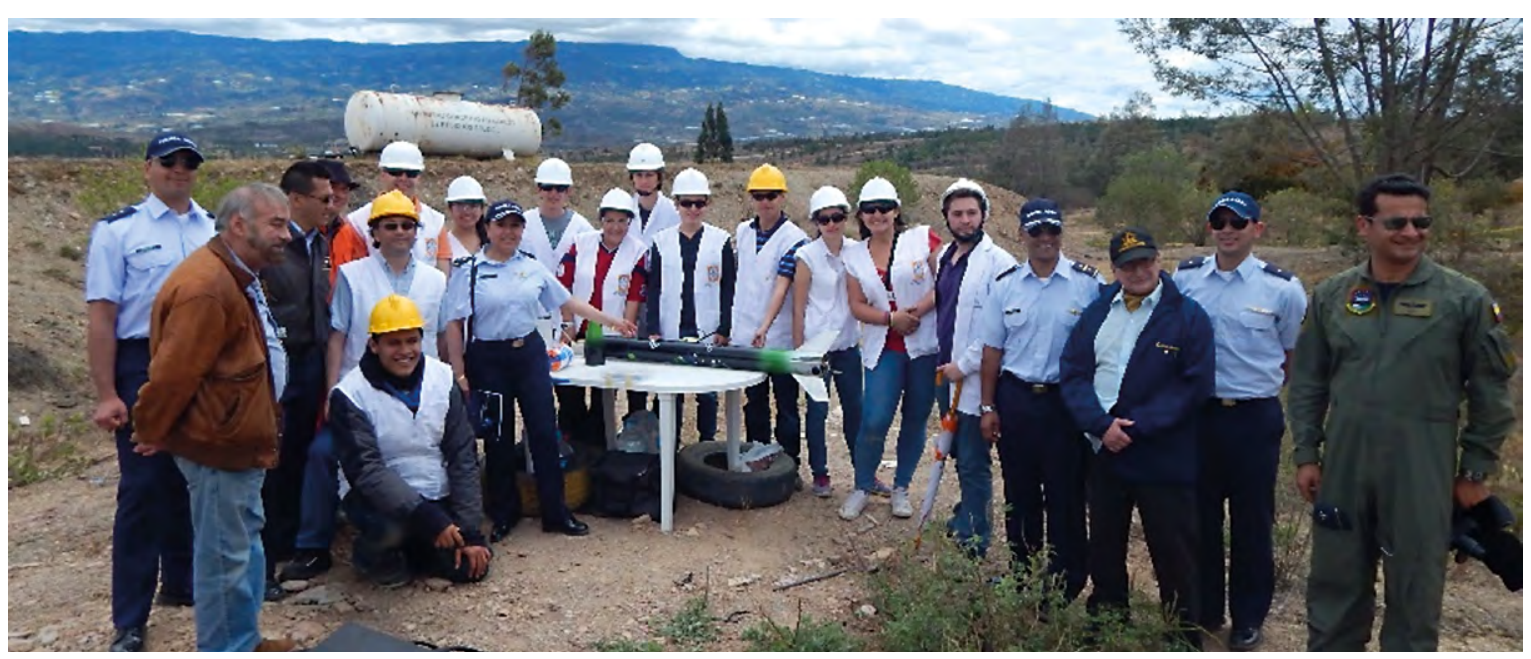

Figura 3. Misión de investigación biológica Séneca IX por parte de GIAS en conjunto con PUA-Uniandes Fuente: los autores.

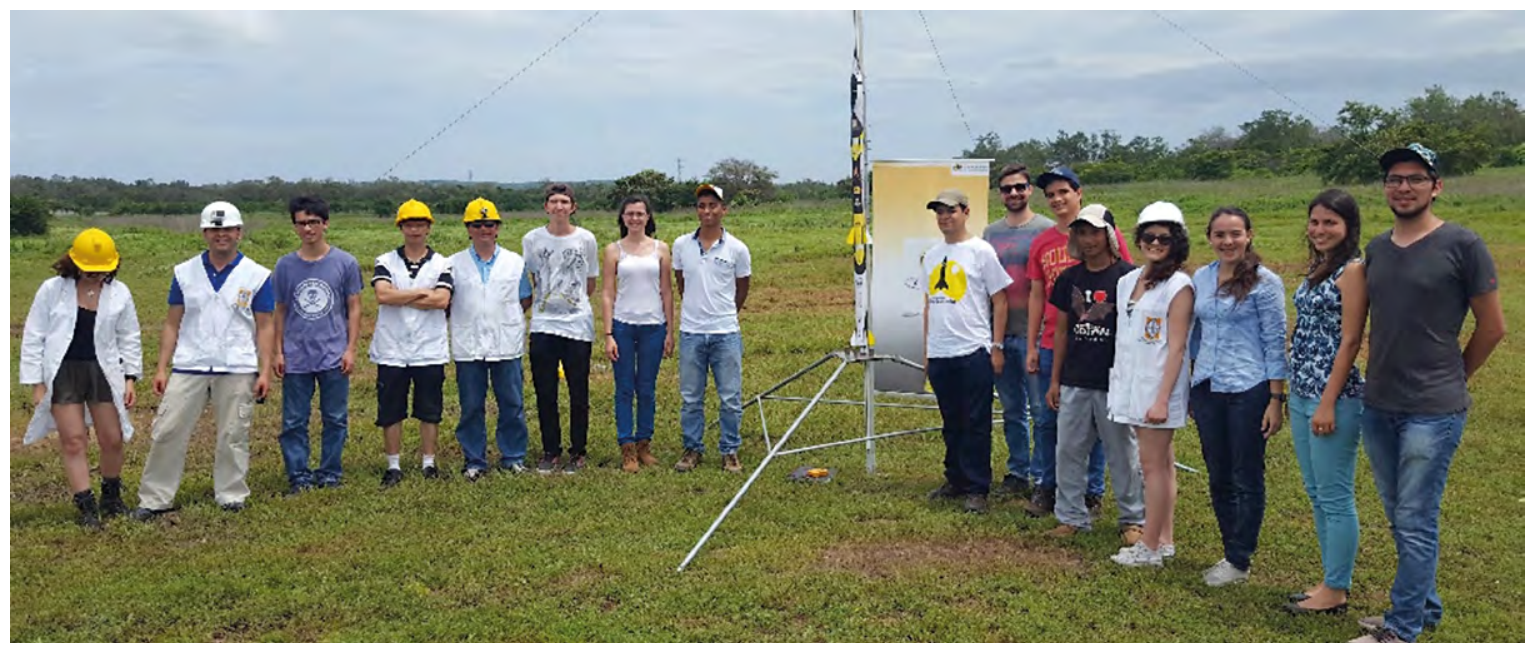

Figura 4. Misión Séneca XI realizada por investigadores de GIAS PUA y Zero G de Uniandinos Fuente: los autores. 


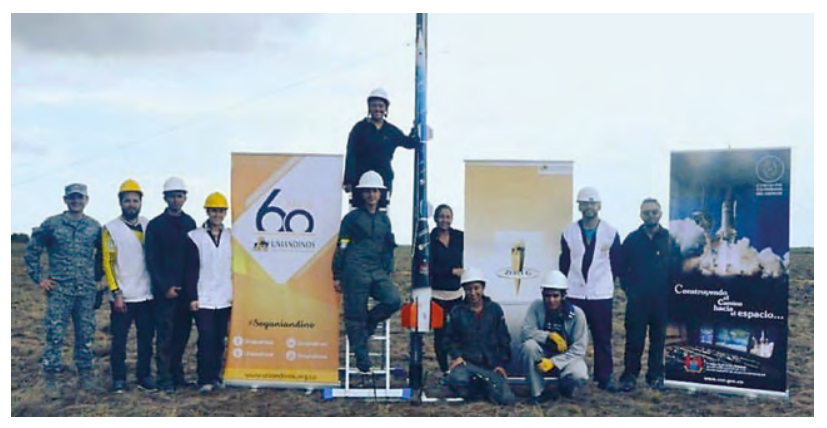

Figura 5. Misión Séneca VII por parte de GIAS junto con PUA Fuente: los autores.

respectivamente, con el fin de adquirir experiencia en el proceso de lanzamiento, recuperación y prueba de cohetes experimentales, ademas de la adquisición y corroboración de conocimientos normativos y reglamentarios, siendo actualmente la única institución en el país que ha logrado una certificación de este tipo.

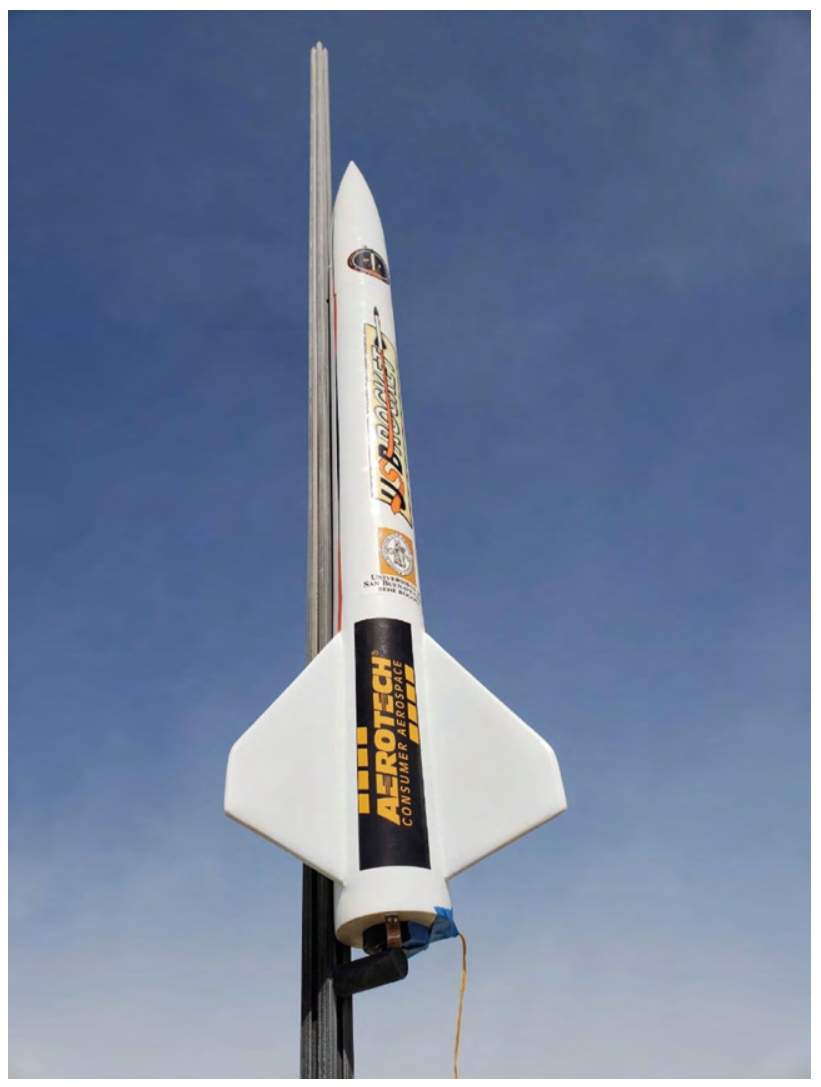

Figura 6. USB-Rocket I para lanzamiento en Nevada, Estados Unidos Fuente: los autores.
Con la experiencia obtenida después de diferentes ejercicios de lanzamiento nacionales con las entidades nombradas, utilizando procedimientos de seguridad basados en diferentes normativas internacionales, y con la experiencia obtenida en Nevada, al utilizar protocolos de seguridad regulados y estandarizados, puede evidenciarse la falta de regulación en el ámbito aeroespacial, debidamente ajustada al contexto colombiano. Esta necesidad estructura el origen del proyecto aquí descrito, el cual en su primera fase estableció los criterios normativos para la experimentación con vehículos tipo cohete. Una vez obtenidos estos criterios normativos, para el año en curso (2020), se busca la caracterización y adaptación al contexto nacional, como segunda fase del proyecto. En esta fase se establecerán unos lineamientos reglamentarios, que permitirán el crecimiento, investigación y divulgación nacional de este campo de la ciencia y la tecnología, además, se facilitará la ejecución y el apoyo para realizar más lanzamientos y experimentos de este tipo, con el uso de procedimientos regulados y aceptados a nivel gubernamental.

\section{Resultados}

\section{Resultados obtenidos en la primera fase del proyecto}

En la fase 1 de este proyecto, con el apoyo de la Fuerza Aérea Colombiana, se obtuvieron resultados completamente provechosos, para efectos de la determinación de procesos normativos preliminares en cohetería colombiana. El primer resultado obtenido en esta fase es la matriz normativa, en la cual se sintetizaron los aspectos a evaluar, enunciados en la metodología para cada una de las normativas internacionales; la matriz normativa es una herramienta de consulta para la determinación, comparación y ubicación de las regulaciones internacionales vigentes. Esta matriz permitirá, en la segunda fase, realizar la caracterización de estas normativas y la adaptación de los diferentes criterios para el contexto colombiano. Este primer resultado se 
ampliará en la segunda fase del proyecto, agregándole información referente a normativas latinoamericanas, con respecto al campo aeroespacial. Esta nueva información se clasificará en los mismos aspectos y se anexarán a la matriz, para que también se tengan en cuenta al momento de la adaptación nacional, con lo cual se obtiene un concepto más próximo de la aplicación de estas regulaciones.

El segundo resultado obtenido en esta fase del proyecto es una matriz de riesgos, realizada bajo la Guía Técnica Colombiana GTC 45, la cual presenta un marco de principios, prácticas y criterios para la identificación de peligros y la valoración de riesgos de seguridad y salud ocupacional (ICONTEC, 2010). Esta matriz está estructurada en cuatro submatrices, la primera compete a todo lo relacionado con el alistamiento, realizado desde el taller de ensamble y al momento de las pruebas de los diferentes elementos; la segunda, comprende aquellos aspectos relacionados con el lanzamiento en el campo de pruebas o bien el campo de disparo; en la tercera, se trata lo referente al embarque, traslado y desembarque del cohete, procesos que pueden ser realizados en zonas francas, aeropuertos 0 terminales de carga, estas tres partes fueron clasificadas según los factores enunciados en la metodología; por último, la cuarta submatriz contiene información de clasificación de los diferentes tipos de peligros, algunos incidentes asociados a la seguridad, a la higiene personal y acciones causantes de peligro. Esta matriz permite al usuario el conocimiento de los posibles peligros y riesgos, que pueden presentarse al momento de realizar experimentación e investigación con vehículos tipo cohete, y además, le proporciona orientación para su mitigación, llevando a cabo los procedimientos de forma adecuada y segura.

\section{Resultados esperados}

Actualmente, este proyecto se encuentra en la segunda fase, como se explicó, y se esperan los siguientes resultados: (1) Realizar la adaptación preliminar de las normativas internacionales al contexto colombiano para su implementación nacional, teniendo en cuenta tanto las normativas expuestas en la primera fase, como las normativas latinoamericanas que se caracterizarán en la segunda fase. (2) Una vez se tengan estos criterios normativos adaptados, se plantearán los protocolos de seguridad para cada una de las fases de la experimentación con vehículos tipo cohete, a saber: logística desde el embarque, traslado y desembarque de los elementos necesarios para la experimentación, alistamiento previo al lanzamiento, cronología de disparo del cohete y la recuperación de este una vez regrese a tierra; estos protocolos irán acompañados de listas de chequeo y verificación, para llevar de forma ordenada y segura el paso a paso de los procedimientos, teniendo en cuenta las diferentes condiciones que dependerán del tipo de cohete. (3) Por último, planteados los protocolos, se le realizarán pruebas de efectividad con el lanzamiento del cohete certificado, perteneciente a la Universidad de San Buenaventura, probando así, la funcionalidad y aplicabilidad de estos procedimientos en el contexto nacional. Estas pruebas serán efectuadas para ultimar el desarrollo de los protocolos y realizar las correcciones que se consideren pertinentes para la conformación final de los mismos. De esta forma, se establecerán, por primera vez, procedimientos reglamentados para el lanzamiento y la experimentación con vehículos tipo cohete, para el uso en el contexto colombiano, claramente de apertura a modificaciones para su constante mejora. Estos protocolos serán usados para proyectar unos lineamientos de normatividad, que serán expuestos a las autoridades aeronáuticas pertinentes, con el fin de consolidar una reglamentación para Colombia en el mediano plazo.

\section{Conclusiones}

Desde la reactivación del semillero GIAS, del Programa de Ingeniería Aeronáutica de la Universidad de San Buenaventura, sede Bogotá, se ha logrado un avance científico e investigativo a nivel nacional bastante amplio, como se puede observar en el desarrollo de este documento. El avance se refleja en la obtención de la primera certificación a nivel nacional de Nivel II, para 
cohetería experimental de alta potencia, otorgado por la TRA; y en la expansión del horizonte de los investigadores nacionales, principalmente en las universidades, generando el interés de alianzas en la búsqueda constante del crecimiento de este sector y encaminados a la adquisición de nuevos procesos de certificación para Colombia.

De la investigación se puede observar que, la falta de normatividad a nivel nacional en el ámbito aeroespacial genera dificultades para su exploración, tales como, la obtención de permisos con los entes reguladores y la importación de tecnologías internacionales, que a su vez generan cierto desinterés y detenimiento en el avance de este campo. Si esta regulación existiera a nivel nacional, permitiría la divulgación y el crecimiento de personal dispuesto a impulsar el sector aeroespacial y el desarrollo nacional en este campo de la ciencia, y propiciaría una mayor interacción de la industria aeroespacial colombiana, con entidades administrativas internacionales para el crecimiento y fortalecimiento de este sector.

De igual forma, se observa que, desde hace aproximadamente sesenta años, Colombia ha sido partícipe de la experimentación e investigación en la industria aeroespacial, y aunque su crecimiento no es comparable con el de los países en los que se basa este proyecto, se ha generado un acervo de conocimiento de suma importancia; además, existen personas y entidades, tanto privadas como públicas, que buscan fortalecer el sector aeroespacial colombiano y, así no esté totalmente consolidado hasta el momento, se han desarrollado avances encaminados a su surgimiento.

Para concluir, se reconoce que otra limitante en el crecimiento del sector aeroespacial, a nivel nacional, es la inyección de capital que se requiere para su desarrollo; aunque el estado y entidades privadas han invertido en el avance científico, esta inversión no ha sido suficiente para explotar el campo aeroespacial que Colombia puede otorgar, lo importante es continuar y en lo posible aumentar la inversión para incrementar la industrialización de este campo, mejorar procesos de manufactura e incentivar la experimentación con fines investigativos, lo que permitirá al sector aeroespacial colombiano estar en constante desarrollo.

\section{Agradecimientos}

Inicialmente, gracias a todos los precursores de esta temática en Colombia, quienes inspiraron a entusiastas e investigadores, como el doctor J. Alejandro Urrego P., a quien se agradece especialmente por ser partícipe de grandes avances a nivel nacional en este campo de la ciencia. A su vez, se agradece al personal de la Universidad de San Buenaventura, sede Bogotá, que de una u otra forma han participado en el desarrollo de esta temática al interior del Programa de Ingeniería Aeronáutica, gracias a cada uno de los estudiantes que han pasado por el semillero GIAS, pues han contribuido y aportado su grano de arena, con proyectos, actividades e investigaciones, al crecimiento del semillero. Finalmente, se agradece a entidades externas estatales y privadas, como lo son la Fuerza Aérea Colombiana y la Universidad de los Andes, quienes compartieron conocimiento y cooperación en conjunto con el semillero para el desarrollo de sus actividades.

\section{Referencias bibliográficas}

ACEMA. (2007). Código de seguridad para experimentación amateur. http://www.acema.com.ar/files/ACEMA-SEG-Expe rimentacion.pdf

ACEMU. (2008). Protocolo de seguridad para la práctica de la cohetería experimental amateur. http://acemu.org/foro/ index.php?topic $=49.0$

Aeropuerto de Medellín Olaya Herrera. (2018). Pacho Cohetes [Archivo en video]. https://aeropuertoolayaherrera.gov. co/pacho-cohetes/

Álvarez, N., Huérfano, J., \& Ojeda, O. (2015, mayo). Diseño e implementación de misión para el lanzamiento de un cohete para tres kilómetros de altura [ponencia]. VIII Congreso Argentino de Tecnología Espacial, Buenos Aires. https://www.researchgate.net/publication/277012130_ DISENO_E_IMPLEMENTACION_DE_MISION_PARA_EL_ LANZAMIENTO_DE_UN_COHETE_PARA_TRES_KILOME TROS_DE_ALTURA

BBC. (2017, octubre 4). Sputnik, el primer satélite que hizo despegar la carrera espacial entre la uRSs y Estados 
Unidos hace 60 años. BBC Mundo. https://www.bbc.com/ mundo/media-41503825

CONPES. (2020). Política de desarrollo espacial: Condiciones habilitantes para el impulso de la competitividad nacional [Documento CONPES 3983]. https://colaboracion.dnp. gov.co/CDT/Conpes/Económicos/3983.pdf

FAA. (2009). Code of Federal Regulations [Title 14 - Aeronautics and Space, Part 101-Moored Balloons, Kites, Amateur Rockets, Unmanned Free, Balloons, and Certain Model Aircraft]. https://www.govinfo.gov/content/pkg/CFR2018-title14-vol2/xml/CFR-2018-title14-vol2-part101. xml\#seqnum101.21

FAA. (2017). A Brief History of the FAA. https://www.faa.gov/ about/history/brief_history/

ICONTEC. (2010). GTC-45: Guía para la identificación de los peligros y la valoración de los riegos en seguridad y salud ocupacional. https://idrd.gov.co/sitio/idrd/sites/default/ files/imagenes/gtc450.pdf

Kelly, B. E. (TRA). (2015). Metal in Rocket Construction. https:// doi.org/10.1017/CBO9781107415324.004

León-Lozada, F. (2018, 3 de diciembre). La Fuerza Aérea de Colombia lanzó FACSAT-1. Latam Satelital. http://latamsa telital.com/la-fuerza-aerea-colombia-lanzo-facsat-1/

Murcia-Piñeros, J. O., \& Portilla-Barbosa, J. G. (2014). Simulación de la trayectoria de un cohete de dos etapas para posicionamiento de un nanosatélite en órbita. Ciencia y Poder Aéreo, 9(1), 107-114. https://doi.org/10.18667/ cienciaypoderaereo.139

NAR. (2000). NAR Safety Officer Training Program [Documento interno NAR]. http://www.nar.org/pdf/TSO.pdf

NAR. (2012a). High Power Rocket Safety Code [Documento interno NAR]. https://www.nar.org/safety-information/hi gh-power-rocket-safety-code/

NAR. (2012b). History. https://www.nar.org/about-nar/history/

NAR. (2012c). Model Rocket Safety Code. https://www.nar.org/ safety-information/model-rocket-safety-code/

NAR S\&T. (2020). Combined CAR / NAR / TRA Certified Rocket Motors List. http://www.nar.org/SandT/pdf/Combined MotorsBylmpulse.pdf

NFPA. (2018a). NFPA 1127. Code for High Power Rocketry. https://www.nfpa.org/codes-and-standards/allcodes-and-standards/list-of-codes-and-standards/ detail?code $=1127$

NFPA. (2018b). NFPA 1122. Code for Model Rocketry. https:// www.nfpa.org/codes-and-standards/all-codes-andstandards/list-of-codes-and-standards/detail?code $=1122$ NFPA. (2019). NFPA Overview. https://www.nfpa.org/overview
NFPA. (2020). NFPA 1225. Standards for Emergency Services Communications. https://www.nfpa.org/codes-and-sta ndards/all-codes-and-standards/list-of-codes-andstandards/detail?code=1225\&year=Proposed Standard \&tab=nextedition

Organismo de Exploración Aeroespacial del Japón, JAXA (2008). Cohetes de Agua. Manual del educador (Trad. Winds, Ltda.). https://2mp.conae.gov.ar/descargas/Materia les\%20/Cohetes_de_Agua-Manual_del_Educador.pdf

Pirateque Bolívar, M. A., \& Sabogal, A. E. (2011). Más que un sueño, una realidad. Historia preliminar de la coheteria en Colombia. Asociación Astronáutica Colombiana Astcol, 1-28. https://www.academia.edu/6643405/ ASOCIACIÓN_ASTRONÁUTICA_COLOMBIANA_ASTCOL_ MÁS_QUE_UN_SUEÑO_UNA_REALIDAD_HISTORIA_ PRELIMINAR_DE_LA_COHETERÍA_EN_COLOMBIA

PUA. (2014). Misión Séneca IX. https://pua.uniandes.edu.co/ doku.php?id=misiones:mision 16

PUA. (2016a). Misión Séneca VII. https://pua.uniandes.edu.co/ doku.php?id=misiones:mision 14

PUA. (2016b). Misión Séneca XI. https://pua.uniandes.edu.co/ doku.php?id=misiones:mision 12

Redacción el tiempo. (1998, 15 de diciembre). Hernán Charry: Un astrósofo que lanza cohetes al espacio. El Tiempo. https://www.eltiempo.com/archivo/documento/MAM853874

Redacción el tiempo. (2007, 28 de septiembre). Alberto Quijano, un pastuso de alto vuelo. El Tiempo. https://www.eltiempo.com/archivo/documento/MAM-2671588

Riveros-Enciso, F., \& Rodríguez-Herrera, L. (2010). Diseño y construcción de un cohete aficionado controlado mediante el accionamiento de una tobera de empuje vertical [Tesis de pregrado]. Universidad Militar Nueva Granada. https://repository.unimilitar.edu.co/bitstream/hand le/10654/3303/RiverosEncisoFelipeAlejandro2010.pdf; jsessionid=C8F9BFED6F9F8194C06DEA1930A832EA?se quence $=2$

Serrato, J. S., \& Ramírez, D. (2018). Ensamble, Puesta a punto y lanzamiento del cohete multietapa Ainkaa Alpha de alcance estratosférico [Tesis de pregrado]. Universidad de San Buenaventura. http://biblioteca.usbbog.edu. co:8080/Biblioteca/BDigital/166523.pdf

TRA. (2004). History. http://www.tripoli.org/History

TRA. (2015a). Range Safety Guidelines [Documento interno TRA]. http://www.tripoli.org/Portals/1/Documents/Safe ty Code/Range Safety Guidelines v1.3.pdf 
TRA. (2015b). Range Safety Officer Guidelines [Documento interno TRA]. http://www.tripoli.org/Portals/1/Docume nts/Safety Code/RSO Guidelines v1.0.pdf

TRA. (2017a). Safety Code for High-Power Rocketry Tripoli Rocketry Association [Documento interno TRA]. http://www. tripoli.org/Portals/1/Documents/SafetyCode/HighPower SafetyCode - 2017.pdf

TRA. (2017b). Tripoli Research Safety Code [Documento interno TRA]. http://www.tripoli.org/Portals/1/Documents/Safe ty Code/ResearchSafetyCode - 2017.pdf

TRA. (2017c). Tripoli Rocketry Association Safe Launch Practices [Documento interno TRA]. http://www.tripoli.org/Por tals/1/Documents/Safety Code/SLP 2017.pdf

UKRA. (2006). About UKRA. http://www.ukra.org.uk/aboutus

UKRA. (2016). UKRA Range Safety Officer. http://www.ukra.org. $\mathrm{uk} / \mathrm{rso}$

UKRA. (2017). Study Guide Safety Officers Examination and Level II Flight Certification Examination. http://www.ukra.org. uk/docs/ukra-l2-exam-guide-v3.0b.pdf

UKRA. (2018). UKRA HPR Certification. http://www.ukra.org.uk/ hprcerts
Urbina-Carrero, J. C. (2017). El espacio, futuro de la Fuerza Aérea Colombiana. Ciencia y Poder Aéreo, 12(1), 202-208. https://doi.org/10.18667/cienciaypoderaereo.572

Urrego, J. (2009). Misión Seneca, Lanzamiento del cohete AINKAA I. Ciencia e Ingeniería Neogranadina, 52(1), 1-5. https://repositorio.uniandes.edu.co/bitstream/handle/ 1992/16673/u371539.pdf?sequence=1

Urrego, J. (2012, septiembre). Estudio de factibilidad de la primera misión colombiana de cohetería experimental con propulsión líquida y alcance estratosférico. Proyecto PUA, Misión SENECA II. Ciencia e Ingeniería Neogranadina, 3, 1-47. https://repositorio.uniandes.edu.co/bitstream/handle/1992/11545/u608089.pdf?sequence=1\&is Allowed=y

Urrego, J., \& Rojas, F. (2010). Misiones de cohetería experimental con propelente sólido: Misión Séneca, Cohete AINKAA 1 / Experimental Rocketry Missions: the Seneca Mission, Ainkaa 1 Rocket. Ciencia e Ingeniería Neogranadina, 20(2), 53. http://ezproxy.uniandes.edu.co:8080/ login?url=http://search.ebscohost.com/login.aspx? direct=true \&db=edssci\&AN=edssci.S0124.81702010000 200004\&lang=es\&site=eds-live\&scope=site 